\title{
The Treatment of Glucocorticoid Steroid Resistant Chronic Inflammatory Disease with Moxa Tar
}

\section{Qian Zeng}

General Practitioner, Medi7 Clinic Bentleigh, Australia

*Corresponding author: Qian Zeng, General Practitioner, Medi7 Clinic Bentleigh, Australia, Tel: 61431552241; E-mail:qianzng@yahoo.com.au

Received date: February 08, 2017; Accepted date: February 21, 2017; Published date: February 24, 2017

Copyright: (c) 2017 Zeng Q. This is an open-access article distributed under the terms of the Creative Commons Attribution License, which permits unrestricted use, distribution, and reproduction in any medium, provided the original author and source are credited.

\begin{abstract}
Currently glucocorticoid steroid is the mainstay of treatment of chronic inflammatory diseases like asthma, COPD, rheumatoid arthritis. But many chronic inflammatory diseases are resistant to glucocorticoid steroid like pulmonary fibrosis, atherosclerosis related diseases, chronic laryngitis and chronic muscle injury. Glucocorticoid steroid has been thoroughly studied and its therapeutic potential has been exhausted, and therefore there is a need to develop new anti-inflammatory drugs or anti-inflammatory therapies. Moxa (Artemisia Argyi) is a plant, its burning smoke or Tar has strong anti-inflammatory property. In this article the author wants to introduce a new way (Moxa Tar) of treating glucocorticoid steroid resistant chronic inflammatory diseases based on some clinical experiments and hypothesis of the author's personal observation. The purpose of this article is to open up a new field and raise researchers' attention to this new field for developing new anti-inflammatory drugs or anti-inflammatory therapies. The methods used in this study are Crossover design, i.e. patients serve as their own control, comparing the clinical symptoms and signs before and after Moxa Tar treatment.
\end{abstract}

Keywords Glucocorticoid steroid; Moxa Tar or Moxa smoke; Moxibustion; Atherosclerosis; COPD (chronic obstructive pulmonary disease).

\section{Introduction}

Glucocorticoid steroid has been widely used to treat a variety of chronic inflammatory diseases, like asthma, COPD (chronic obstructive pulmonary disease), rheumatoid arthritis, inflammatory bowel diseases, since it was invented in 1948. But many chronic inflammatory diseases remain resistant to glucocorticoid steroid like chronic atrophic gastritis, chronic muscle injury, ligament injury, soft tissue injury, thromboangiitis obliterans (Buerger's disease), chronic laryngitis, pulmonary fibrosis, multiple sclerosis, systemic progressive sclerosis, atherosclerosis related diseases and so on.

\section{Methods}

Glucocorticoid steroid has been thoroughly studied and its therapeutic potential has been exhausted. In order to treat glucocorticoid steroid resistant chronic inflammatory diseases, we must develop new anti-inflammatory drugs or therapies.

In this article, the author wants to introduce a new way (Moxa Tar) of treating glucocorticoid steroid resistant chronic inflammatory diseases through his personal experience in fighting against them.

The author suffered from acute laryngitis secondary to flu in 2000 . His acute laryngitis was treated with oral Prednisolone and his condition quickly improved. But after three episodes of improvement and deterioration of the acute laryngitis, his condition progressed to chronic laryngitis [1]. The author was advised by his family doctor that his chronic laryngitis would never be cured because the underlying mechanism of chronic inflammation was different from that of acute inflammation and glucocorticoid steroid was not a cure for chronic inflammation.
In desperation, the author turned to Traditional Chinese Medicine for help. He tried 15 different therapies, including herbal therapies and heat therapy, but none of them worked until he found moxibustion. Moxibustion is a therapy and has two thousand and five hundred years history in China. It is highly regarded by traditional Chinese herbalists. They claim that it can treat hundreds of different diseases including many intractable cases. But it is little known to Western society [2]. In this therapy, an herb called Artemisia Argyi, or Moxa or Chinese mugwort is being used. It is to grind dried Moxa up to fluff and further process it into a cigar-shaped stick or a cone. Practitioners burn this Moxa stick to heat certain acupuncture points on the patient's skin or put the Moxa cones on the acupuncture points and burn them to treat various diseases.

Chinese herbalists use the theory of Traditional Chinese Medicine to explain the therapeutic mechanism of moxibustion. They claim that the burning Moxa produces a special radiating heat which can penetrate the skin, give extra energy to the body, warm the blood and open the body's blocked meridian lines [3] to improve the blood flow to treat diseases. It is very difficult for Western doctors to understand these theories and moxibustion is often not accepted by practitioners of Western medicine. To date Western medicine circles have ignored it as non-scientific at best and superstitious at worst [2].

The author decided to try the therapy. He applied Moxa smoke to the skin above the larynx by waving burning Moxa stick towards larynx for 15 to 20 minutes once a day, or alternatively had 3 to 8 inhalations of Moxa smoke once a day (both methods have equal therapeutic effect. Moxa smoke becomes Moxa Tar when it reaches to the skin above the larynx or mucosa in the larynx), continued for 15 days as a course. There was 3 -day interval between two courses $[4,5]$.

After two courses of treatment with moxibustion, the pain, dryness and swelling in the larynx started to ameliorate. With further 5-course treatment, all the pain, dryness and swelling in the larynx completely 
disappeared and the author was able to talk normally again. The chronic laryngitis had been cured.

The author's experience proves that chronic inflammation could be cured by moxibustion. The author proposes a different explanation from that of Traditional Chinese Medicine of the mechanism of the therapeutic effect of moxibustion according to his personal experience [1,6-9]. The author thinks that the curative effect of moxibustion is not due to the heat produced by the burning Moxa stick, but is due to the Moxa Tar (Moxa smoke becomes Moxa Tar when it reaches to the skin or vocal cord) produced by the burning Moxa stick. The heat required in moxibustion is only to open the hair follicles and dilate blood vessels to improve local blood circulation, facilitating the delivery of the Moxa Tar into the tissue. The heat produced by burning Moxa per se does not have anti-inflammatory effect or special therapeutic effect just as the heat produced by other methods. Without the Moxa Tar (or Moxa smoke), moxibustion does not have any therapeutic effect.

The author did experiments, using foil to cover the skin above the larynx, and then applied Moxa smoke to the foil. The heat produced by burning Moxa stick could penetrate the foil to heat the skin and the tissue below the foil, but the Moxa Tar could not reach to the skin. After many treatments with moxibustion, there was no any improvement in the symptoms of the chronic laryngitis [1]. The other experiment was to dissolve Moxa smoke in water and to apply the water to the skin above the larynx of chronic laryngitis patients and to eczematous lesions on the skin of eczema patients. The results were that both of these diseases were cured. This experiment proved that without the heat, Moxa Tar still had anti-inflammatory effect.

These two experiments demonstrate that the active antiinflammatory ingredients are present in the Moxa Tar and the heat alone has no anti-inflammatory effect.

The prolonged severe chronic laryngitis caused severe tissue damage to the vocal cord of the author. Every time he had flu, the flu viruses (flu virus is a strong $\mathrm{T}$ cell activator) triggered the chronic laryngitis. Without moxibustion treatment, the inflammation would last forever.

From November 2000 to June 2016, the author had ten episodes of chronic laryngitis, all triggered by flu viruses. Fortunately all ten episodes of chronic laryngitis had been cured by moxibustion. The author's vocal function was perfectly normal between episodes of his chronic laryngitis [10].

The author also used similar therapy to treat more chronic laryngitis patients.

Selection of patients: random.

Number of patients: 25

Gender of patients: female: 11 , male: 14

The age of patient: 26 to 85 years old

Location of patients: 3 in Australia, 22 in China

\section{Criteria of diagnosis}

Diagnosis was based on clinical symptoms and signs (laryngoscopy is not reliable for diagnosis of chronic laryngitis, laryngoscopy examination could be normal but patients still had voice fatigue and symptoms in the larynx).

All patients had hoarse voice, pain, dryness and heaviness in the larynx and voice fatigue for more than 6 months without improvement.

\section{Previous treatment:}

All patients had oral Prednisolone treatment from $25 \mathrm{mg}$ daily to 50 $\mathrm{mg}$ daily for 7 days to 30 days. None of them had response to Prednisolone treatment.

\section{Current treatment}

Method 1: Applying Moxa smoke to the skin above the larynx for 15 to 20 minutes one session a day or alternatively having 3 to 8 inhalations of Moxa smoke one session a day (both methods have equal therapeutic effect, one delivers Moxa Tar to larynx through skin absorption, another directly deposits Moxa Tar to the surface of vocal cord), 15 days is a course. There is a 3-day interval between two courses. 22 patients received this therapy $[11,12]$.

Method 2: Applying Moxa-smoke-dissolved-water to the skin above the larynx 2 to 3 times a day, 15 days is a course, and there is 3-day interval between two courses. 3 patients received this therapy.

\section{Duration of treatment}

13 patients had 2 courses of treatment of method 1.

9 patients had 4 courses of treatment of method 1.

3 patients had 4 courses of treatment of method 2 .

None of the 25 patients were allowed to take spicy food, smoking or drinking alcohol during their treatment.

\section{Evaluation of therapeutic effect}

Evaluation of therapeutic effect is based on the clinical symptoms, signs and vocal function.

13 patients showed improvement in the pain, dryness and heaviness in the larynx and hoarse voice after 1 course of treatment of method 1. When they completed the $2^{\text {nd }}$ course of treatment of method 1 , all the pain, dryness and heaviness in the larynx and hoarse voice disappeared and could talk normally without any voice fatigue.

9 patients had improvement in the pain, dryness and heaviness and hoarse voice after 2 courses of treatment of method 1 . When they completed 4 courses of treatment of method 1, there was no pain, dryness or heaviness in the larynx, had no hoarse voice and could continue to talk for hours without any voice fatigue.

3 patients had improvement in the pain, dryness and heaviness in the larynx and hoarse voice after 2 courses of treatment of method 2 . When they completed 4 courses of treatment of method 2, they did not have any pain, dryness or heaviness in the larynx or hoarse voice and could talk as much as they wanted without any voice fatigue.

Followed up: Followed up for 3 months, no relapse.

The criteria of curing of chronic laryngitis: complete disappearance of symptoms (pain, dryness and heaviness in the larynx and sign (hoarse voice) of chronic laryngitis and returning to normal vocal function, i.e. talking without voice fatigue. The latter is the most accurate and important indicator of the efficacy of assessment for treatment of chronic laryngitis. If a tiny inflamed tissue left in the larynx, the chronic inflammation will rapidly flare up when the patient starts to talk.

No relapse for at least for 3 months.

According to the above criteria, the author considers all 25 chronic laryngitis patients have been cured by Moxa Tar therapy. 
Page 3 of 9

\section{Cure rate: $100 \%$}

The above facts prove that the anti-inflammatory effect of the Moxa Tar can be reliably reproduced on different patients. The underlying mechanisms that sustain the chronic inflammation can be conquered by Moxa Tar.

Chronic laryngitis is typical chronic non-specific inflammation. The author draws a conclusion from this clinical experiment that Moxa Tar is a cure of chronic non-specific inflammation.

It is of great significance. Though chronic laryngitis is not a life threatening disease, it represents the incurability of chronic selfperpetuating inflammation. Chronic self-perpetuating inflammation is an important basic issue in pathology. Its importance is that it is involved in the pathogenesis of many chronic inflammatory diseases and it cannot be cured by known anti-inflammatory drugs. Curing chronic laryngitis means that chronic self-perpetuating inflammation can be cured. Its significance is far greater than curing of one particular chronic inflammatory disease. As many chronic inflammatory diseases share the same underlying mechanism as that of chronic laryngitis, the author hypothesized that Moxa Tar could be used to treat other glucocorticoid steroid resistant chronic inflammatory diseases [13].

In chronic inflammation, the prolonged inflammation causes tissue damage. The inflammation becomes self-perpetuating i.e. the inflammation will last forever even when the initiating stimuli are eliminated. Lymphocyte (mainly $\mathrm{T}$ cell) infiltrate is a character of chronic inflammation. There are complicated intercellar reactions between $\mathrm{T}$ cells and other cells, like macrophages (in chronic laryngitis, COPD ...), neutrophils (in Buerger's disease, COPD), endothelial cells (atherosclerosis) and eosinophils (in asthma) in chronic inflammation. In these intercellar reactions, activated macrophages, neutrophils, eosinophils and endothelial cells produce interleukin 1 [2] and other cytokines to activate $\mathrm{T}$ cells, in turn, activated $\mathrm{T}$ cells secret interferon-gamma, interleukin 8 and other cytokines to activate macrophages, neutrophils, eosinophils, and endothelial cells, the results are positive feedback loops between these cells that fuel and sustain chronic inflammation.

Once these cycles of intercellar reactions are established, no medication can terminate these intercellar reactions.

The therapeutic value of Moxa Tar is that it can disrupt these cycles of intercellar reactions and therefore it can cure self-perpetuating chronic inflammation.

As previously mentioned, many chronic inflammatory diseases could have the same or similar underlying mechanisms. The author decided to generalize the application of Moxa Tar to treat different chronic inflammatory diseases.

\section{Treatment}

\section{Treatment of glucocorticoid steroid resistant eczema}

Glucocorticoid steroid is the mainstay of treatment of eczema.

But 1 or 2 of 10 eczema patients do not respond to glucocorticoid steroid. In those steroid resistant eczema patients, the erythema, exudates, blisters and itchiness could last for many months or years.

The author treated 22 steroid resistant eczema patients with Moxa Tar and Moxa-smoke- dissolved-water.

\section{Selection of patients}

Eczema patients who do not respond to glucocorticoid steroid treatment.

Number of patients: 22

Gender of patients: female: 17 , male: 5

The age of patients: 1 to 60 years old.

Location of patients: 4 in China, 18 in Australia

\section{Diagnosis}

Patients had recurrent or continuous severe skin itchiness and typical eczematous skin lesion, i.e. blisters, exudates, erythema and skin cracks for at least 6 months.

\section{Previous treatment}

All patients had eczema for more than 6 months and received treatment of glucocorticoid cream or ointment for at least 2 months accumulatively, but had no or minimal response ( eczema symptoms and signs could not be effectively suppressed by glucocorticoid steroid therapy) to the treatment.

\section{Current treatment}

\section{The method of treatment}

Method 1: Applying Moxa smoke to eczematous lesions for 15 minutes, one session a day. 15 days is a course. There is a 3-day interval between two courses. 12 patients received this therapy.

Method 2: Applying Moxa-smoke-dissolved-water to eczematous lesions 2 to 3 times a day. 15 days is a course. There is a 3-day interval between two courses. 10 patients received this therapy.

\section{Duration of treatment and assessment of the efficacy of treatment}

Assessment was based on the clinical symptom (itchiness) and signs (exudates, blisters, erythema and skin cracks) of eczema.

\section{Assessment of the efficacy of method 1}

5 patients' symptom and sings of eczema completely disappeared after 1 course of treatment of method 1 .

6 patients' symptom and sings of eczema completely resolved after 2 courses of treatment of method 1.

1 patient had no change in his symptom and sings of eczema after 3 courses of treatment of method 1.

Assessment of the efficacy of method 2:

2 patients' symptom and signs of eczema completely disappeared after 1 course of treatment of method 2.

6 patients' symptom and signs of eczema completely resolved after 2 courses of treatment of method 2.

2 patients had no improvement in their symptom and signs of eczema after 3 courses of treatment of method 2.

11 of 12 eczema patients who received method 1 treatment were cured (this episode of eczema was cured, eczema often relapse) by Moxa Tar therapy.

Success rate: $91 \%$ 
1 of 12 eczema patients who received method 1 treatment did not response to Moxa Tar therapy.

\section{Failure rate: $9 \%$}

Followed up for 2 years, 6 of 11 cured patients by method 1 relapsed from 5 months to 2 years after the discontinuation of treatment, retreated with the same therapy for 1 to 2 courses, their eczema were cured again.

8 of 10 eczema patients who received treatment of method 2 were cured (this episode) by Moxa-smoke- dissolved-water therapy.

Success rate: $80 \%$

2 of 10 eczema patients who received treatment of method 2 did not response to Moxa- smoke-dissolved-water therapy.

\section{Failure rate: $20 \%$}

Followed up for 2 years, 5 of 8 eczema patients cured by method 2 relapsed from 6 months to 20 months after stopping treatment.

Relapsed patients were treated again with Moxa-Tar-dissolvedwater for 1 to 2 courses, all patients were cured again.

The above cases indicate that Moxa Tar can effectively treat glucocorticoid resistant eczema.

\section{Treatment of COPD with Moxa smoke}

COPD is a chronic inflammatory lung diseases caused by various stimuli like smoking, air pollution and exposing to noxious agents.

From the point of view of pathology, the inflammatory cell types involved in the pathogenesis of COPD are alveolar macrophages, neutrophils and T cells. Like chronic laryngitis, there are also cycles of bidirectional intercellar reactions in COPD. The bidirectional intercellar reactions in COPD are between $\mathrm{T}$ cells and alveolar macrophages and $\mathrm{T}$ cells and neutrophils [14].

As Moxa Tar can cure the inflammation in chronic laryngitis, the author inferred that Moxa smoke (Moxa Tar) should be able to treat and might cure the inflammation in COPD too.

\section{Treatment of COPD with Moxa Tar (Moxa smoke)}

Number of patients: 25

Gender of patient: female: 5 , male: 20

Age of patients: 50-88 year old

Location of patients: 24 patients were in China, 1 patient was in Australia.

Selection of patients: no active respiratory tract infection, cessation of smoking.

22 patients had a history of cigarette smoking for more than 10 years.

3 patients had no history of smoking

All cigarette smokers had stopped smoking for at least one year before receiving the inhalation therapy of Moxa smoke.

Current medications: SABA, LABA, LAMA and or ICS.

\section{Classification of patients}

All 25 patients: $\mathrm{FEV}_{1} / \mathrm{FVC}<70 \%$

3 patients: $\mathrm{FEV}_{1}<30 \%$ predicted, had severe dyspnoea and cyanotic lips at rest, had no productive cough, classified as very severe emphysema patients.

10 patients: $30 \%$ predicted $<\mathrm{FEV}_{1}<40 \%$ predicted, had dyspnoea on minimal exertion with or without chronic productive cough, classified as severe patients.

12 patients: $\mathrm{FEV}_{1}=40-59 \%$ predicted, had dyspnoea on moderate exertion with or without cough and/or sputum, classified as moderate severe patients.

22 patients: diagnosed as emphysema by CT scan

3 patients: had dyspnoea and productive cough for more than three consecutive months in more than two consecutive years without imaging evidence of emphysema, diagnosed as chronic obstructive bronchitis.

These 3 patients: $40 \%$ predicted $<\mathrm{FEV}_{1}<59 \%$ predicted, classified as moderate severe chronic obstructive bronchitis patients.

All 25 patients still had mild to severe COPD symptoms (shortness of breath on exertion) with treatment of inhaled bronchodilators and /or ICS before receiving inhalation therapy of Moxa smoke.

All 25 patients had moderate to severe COPD symptoms (from breathless walking on level ground to severe dyspnoea at rest) after one or two days of discontinuation of inhaled bronchodilators and/or ICS [15].

\section{Pre-conditions for receiving treatment}

1. No evidence of active respiratory tract infection (no fever, no yellow or green colour sputum)

2. If patients did have active respiratory tract infection, they must be treated with antibiotics to clear the infection before starting the inhalation treatment.

3. Stop smoking.

\section{Treatment}

\section{Method of treatment:}

3-8 inhalations of Moxa smoke per session a day

15 days as a course

Total treatments: $2-4$ courses (there is a 3-5 day break between courses)

Alternative treatment (for those who cannot cope with smoke inhalation)

It is to apply the Moxa smoke to the front and back chest except the precordial area by waving a burning Moxa stick for 7 minutes to 10 minutes, one session a day.

15 days as a course

Total treatments: 2 courses to 4 courses (there is a 3-5 day break between courses)

23 patients: treated with inhalation therapy of Moxa smoke. 
Page 5 of 9

2 patients: treated with alternative therapy (they could not cope with smoke inhalation)

Normally when patients start a new therapy, they should discontinue with the previous therapy, but COPD patients would have severe shortness of breath if they suddenly stop inhaled bronchodilators (SABA, LABA and LAMA) and or ICS, therefore these patients were allowed to keep their previous treatment with inhaled bronchodilators and or ICS when they started the inhalation therapy of Moxa smoke or the alternative therapy if they had severe shortness of breath without inhalation of bronchodilators and or ICS. They continued with this co-treatment until the inhalation therapy or alternative therapy started to take effect i.e. they had less shortness of breath, compared with when only using bronchodilators and or ICS [16].

Then they stopped bronchodilators/ICS completely, but continued with inhalation therapy of Moxa smoke until their COPD symptoms completely disappeared. The "wash out" period of the effect of SABA, LABA, LAMA and ICS is one day.

All 3 very severe patients and 10 severe patients had co-treatment initially.

7 of 12 moderate patients also had co-treatment until the inhalation therapy started to take effect.

5 of 12 moderate patients stopped previous treatment of inhaled bronchodilators and or ICS one day before they started inhalation therapy of Moxa smoke. They managed to cope with shortness of breath after discontinuation of inhaled bronchodilators and or ICS by reducing physical activity.

\section{Evaluation of the Therapeutic Effects of Moxa Tar treatment}

Clinical therapeutic effects were evaluated according to COPD symptoms and values of spirometry.

$3 / 25$ patients ( 1 chronic obstructive bronchitis patient, 2 moderate emphysema patients) had no improvement in their COPD symptoms ( shortness of breath) and spirometry values after having one course (15 days) treatment with the inhalation of Moxa smoke and refused to continue treatment with this therapy.

22/25 patients showed improvement in breathlessness and or cough with or without sputum after 1 or 2 courses of treatment with inhalation of Moxa smoke or alternative therapy.

20 of 22 improved Patients had co-treatment initially; at this stage they stopped treatment with bronchodilators or ICS but continued treatment with the inhalation therapy of Moxa smoke or the alternative therapy for another 1 or 2 courses until symptoms free.

2 of 22 improved patients continued with mono-therapy (only inhalation of Moxa smoke) for another course until symptoms free.

The lips cyanosis of 3 very severe emphysema patients has disappeared after 4 courses of treatment with inhalation therapy.

\section{The criteria of symptoms free}

1. No breathlessness on moderate exertion for moderate severe and severe patients

2. No breathlessness on walking level ground for very severe patients

3. No productive cough
4. Do normal daily life activities without limitation

Comparison of spirometry values of 22 symptoms free patients before and after Moxa Tar treatment

3 very severe and 10 severe emphysema patients: No change in spirometry value before and after Moxa Tar treatment.

7 moderate severe emphysema patients:

Before Moxa Tar treatment: $\mathrm{FEV}_{1} / \mathrm{FVC}<70 \% ; \mathrm{FEV}_{1}=40 \%$ to $59 \%$ predicted.

After Moxa Tar treatment: $\mathrm{FEV}_{1} / \mathrm{FVC}<70 \% ; \mathrm{FEV}_{1}=50 \%$ to $70 \%$ predicted.

2 moderate severe chronic obstructive bronchitis patients:

Before Moxa Tar treatment: $\mathrm{FEV}_{1} / \mathrm{FVC}<70 \% ; \mathrm{FEV}_{1}=40 \%$ to $59 \%$ predicted.

After Moxa Tar treatment: $\mathrm{FEV}_{1} / \mathrm{FVC}<70 \% ; \mathrm{FEV}_{1}>80 \%$ predicted.

All these 22 patients became symptoms free after 2 to 4 courses of treatment with Moxa Tar. They could do their normal daily life activities without limitation and did not need any further treatment with SABA, LABA, LAMA and or ICS.

These patients were followed up from 6 months to 2 years, no relapse. All 22 patients kept symptoms free.

Spirometry tests were repeated for these 22 symptoms free patients 6 months to 2 years after Moxa Tar treatment, the spirometry values of all 22 patients were the same as that when they had just finished Moxa Tar treatment.

At clinical level, Moxa Tar therapy show some curative effect for COPD patients, i.e., after a period of treatment with Moxa Tar, 22 of 25 COPD patients kept symptoms free without any further treatment.

Symptoms free rate is $88 \%$.

3 patients had no improvement in their COPD symptoms or spirometry values after one course (15 days) treatment with inhalation of Moxa smoke.

\section{Failure rate: $12 \%$}

Self-comparison of these 25 patients showed big difference in their COPD symptoms before and after Moxa Tar treatment.

Before Moxa Tar treatment, all 25 patients needed daily treatment with inhaled bronchodilators and or ICS, but they still had mild to severe shortness of breath and or cough with or without sputum; without treatment with inhaled bronchodilators or ICS, all these 25 patients had moderate to severe dyspnoea and or cough with or without sputum, their daily life activities were severely affected by COPD.

After Moxa Tar treatment, 22 of 25 patients kept symptoms free without any further treatment.

The difference of COPD symptoms of these 22 of 25 patients before and after Moxa Tar treatment is significant [1].

As Moxa Tar has been proved by many moxibustion practitioners that it can cure many different chronic inflammatory diseases (chronic laryngitis, chronic muscle injury, chronic pelvic inflammatory disease, chronic atrophic gastritis, thrombophlebitis obliteran), the author presumes that the disappearance of COPD symptoms for these 22 of 25 patients who received Moxa Tar treatment could be due to curing of 
the inflammation in the lungs and respiratory tract. This would correct the ventilation perfusion mismatch in the lungs of these patients and significantly increase the efficiency of gas-exchanging in the lungs, resulting in COPD symptoms free in these patients though there is no change or only slightly change in the results of lung function tests. But this presumption needs to be validated by more objective tests, like diffusing capacity for carbon monoxide, bronchoalveolar lavage for counting inflammatory cells, or bronchoscopic biopsy for pathology before and after treatment.

The author assumes that the reason that there is no improvement in the Spirometry values of severe and very severe patients after treatment with Moxa Tar is that the tissue damage in the lungs of these patients cannot be reversed though the inflammation could be cured by Moxa Tar. Curing of inflammation in the lungs can stop the progression of COPD but cannot reverse the tissue damage.

The author needs to point out that in order to cure the inflammation in COPD, the Moxa smoke (or Moxa Tar) must be delivered to all the inflamed lung tissues to eradicate the inflammation. If using traditional moxibustion to treat COPD patient by applying Moxa smoke to only a few acupuncture points on the skin closest to the lungs, the Moxa Tar can only be delivered to parts of the inflamed lung tissues through the skin absorption. In that case, the inflammation in the lung tissues cannot be eradicated by Moxa Tar and therefore the patient who receives the treatment only shows temporary improvement in his or her COPD symptoms during the treatment and a few months after the cessation of the treatment, but cannot be cured by moxibustion as there is still residual inflammation in the lung tissues which can spread back to the cured lung tissues, the symptoms of COPD can flare up again [15]. That is why the author invented the inhalation therapy of Moxa smoke and modified the moxibustion therapy by applying Moxa smoke to the whole front and back of the chest to treat COPD.

Treatment of chronic muscle injury, ligament injury, soft tissue injury and chronic bursitis with Moxa smoke (Moxa Tar).

Chronic muscle injury, ligament injury, soft tissue injury and chronic bursitis are very common conditions at clinical practicing. They may respond to local glucocorticoid steroid injection at the early stage of these conditions. But they are resistant to steroid treatment when they progress to chronic stage and there are no therapies that could cure these chronic inflammatory conditions. The underlying mechanisms of these chronic injuries are chronic inflammation.

The author treated 65 cases (50 patients in Australia, 15 patients in China) of these chronic conditions with Moxa Tar, including chronic ankle injury, chronic shoulder injury, chronic back muscle injury, various soft tissue injuries and chronic bursitis in knee, shoulder and hip [15]. All these patients had tried many different therapies (include local steroid injection) before they received Moxa Tar treatment, but none of their conditions had improved.

The author treated these chronic injuries and bursitis by applying Moxa smoke directly to the painful and swollen area for 15 minutes to 20 minutes, one session daily, 15 days a course, there was a 3-day interval between courses. The Moxa smoke became a brown colour Tar, i.e. Moxa Tar when it reached to the skin of treated patients. Patients were advised to keep this Moxa Tar on the skin as long as possible (at least for 10 hours) after treatment with Moxa smoke.
60 of 65 patients of chronic muscle, ligament or soft tissue injuries and chronic bursitis were cured after 1 to 4 course of treatment with Moxa smoke.

\section{The criteria of cure}

Complete disappearance of pain, swelling and redness for 3 months; restoration of normal function (The injured muscles, ligaments or soft tissues can bear the intensity of normal physical activities).

\section{Cure rate: $92 \%$.}

5 of 65 patients had no improvement in the pain, swelling or redness in the injured muscles, ligaments or soft tissue after 2 courses of treatment with Moxa Tar.

\section{Failure rate: $8 \%$.}

The author believes that Moxa Tar can cure chronic muscle, ligament and soft tissue injuries because it cures the chronic inflammation in these chronic conditions.

An important point is that patients must have good rest during treatment. Without rest of the injured muscle, ligament or soft tissue, the efficacy of Moxa smoke will be greatly affected.

\section{Treatment of chronic atrophic gastritis}

$90 \%$ of chronic atrophic gastritis is caused by Helicobacter pylori infection. But clearance of Helicobacter pylori infection does not necessarily cure chronic atrophic gastritis.

\section{Treatment of atrophic gastritis}

Number of patients: 15

Location of patients: 5 in Australia, 10 in China.

Gender of patients: female: 10 , male: 8

Selection of patients: random.

\section{Diagnosis}

All patients had epigastric pain and or discomfort for more than one year and were diagnosed as chronic atrophic gastritis by gastroscopy.

Past history: 13 of the 15 patients had history of Helicobacter pylori infection and the infection had been cleared with antibiotics treatment. They however still had chronic epigastric pain and discomfort and had been treated with proton pump inhibitor, but their symptoms did not respond to proton pump inhibitor.

The other 2 of 15 patients had no history of Helicobacter pylori infection. They had been treated with proton pump inhibitor but no response.

\section{Current medication}

All 15 patients stopped proton pump inhibitor.

Method of treatment: applying Moxa smoke to epigastric area for 15 minutes, one session a day, 15 days is a course. There is a 3-day interval between two courses.

\section{Duration of treatment and assessment of efficacy of treatment}

5 patients had 1 course of treatment.

Their epigastric pain and or discomfort have completely disappeared after the treatment. 
10 patients had 2 courses of treatment.

8 of 10 patients' epigastric pain and or discomfort have completely disappeared after the treatment.

2 of 10 patients had no improvement in their epigastric pain and or discomfort after the treatment.

Followed up all 15 patients for 6 months, no relapse.

\section{Success rate: $86.6 \%$}

\section{Failure rate: $13.4 \%$}

10 of the 13 pain free patients had repeated gastroscopy after Moxa Tar treatment and the results of 6 of them did not show chronic gastritis compared with their previous positive findings of gastroscopy.

The above data indicate that Moxa Tar can effectively treat chronic atrophic gastritis.

A moxibustion practitioner reports that in a clinical trial of 160 patients of chronic atrophic gastritis with moxibustion treatment, $85 \%$ of patients' epigastria pain and/or discomfort resolved; 65\% of the 160 patients' repeated gastroscopy showed disappearance of chronic gastritis.

\section{Treatment of atherosclerosis related diseases}

Atherosclerosis is the most important chronic inflammatory pathologic process because atherosclerosis related diseases (heart attack, stroke, peripheral vascular disease) are responsible for about $20 \%$ of total death in the world.

The most accepted theory (hypothesis) of atherogenesis is the inflammatory reaction to injury theory. This theory (hypothesis) considers that atherosclerosis is a chronic inflammatory response of the vascular wall to a variety of insults, including endothelial injury, lipid accumulation and oxidation, and thrombosis. Inflammatory cells and mediators are involved in the initiation, progression, and the complications of atherosclerotic lesions. Inflammation is intimately linked with atherosclerotic plaque formation and rupture.

Currently, prevention and treatment of atherosclerotic related diseases are focused on control of the risk factors, like blood pressure, dyslipidaemia, hyperglycaemia and smoking. No drug can control or reverse the underlying biochemical process of atherosclerosis. The underlying biochemical process of atherosclerosis is an inflammatory process, but this inflammation does not respond to any antiinflammatory drugs $[12,14]$.

There are activated macrophages and T cells in atherogenesis. T cell always plays an important role in sustaining chronic inflammation in various chronic inflammatory diseases. The author hypothesizes that like in many other chronic inflammatory diseases, activated macrophages and $\mathrm{T}$ cells may form a cycle of bidirectional reactions between them (i.e. macrophages secret interleukin 12 to activate $\mathrm{T}$ cells, in turn, $\mathrm{T}$ cells secret interferon gamma to activate macrophages) to fuel and sustain the chronic inflammation in atherosclerosis. As Moxa Tar has unique and potent anti-inflammatory effect and has successfully treated and cured both macrophages and $\mathrm{T}$ cells involved chronic inflammatory diseases, like chronic laryngitis, chronic muscle injury, chronic inflammatory pelvic disease..., the author hypothesizes that Moxa Tar can effectively treat the chronic inflammation in atherosclerosis.
On the other hand, in atherosclerosis, the activated macrophages secrete fibrogenic growth factors, reactive oxygen species, TNF alpha and interleukin I, all these factors are involved in atherogenesis. If the inflammation in atherosclerosis can be suppressed or terminated by Moxa Tar, it may lead to the stopping of the growth or regression of the atheroma and will definitely reduce the risk of thrombosis in the artery surface (TNF alpha and interleukin I upregulate prothrombotic molecules, like Von Willebrand factor, tissue factor and plasminogen activator inhibitor in the endothelium of artery; changing the artery surface from an anti-thrombotic state to a prothrombotic).

There are some observational evidences that support the author's hypothesis that Moxa Tar could treat atherosclerosis.

\section{Treatment of angina pectoris}

Selection of patients: random

Number of patients: 15

Gender of patients: female: 8 , male: 7

Age of patients: 52 to 88 years old

Location of patients: 12 in Australia, 3 in China.

\section{Diagnosis}

All 15 patients had more than one year history of recurrent typical ischemic chest pain, i.e. left upper chest pain, often triggered by physical activity, lasted from a few minutes to 30 minutes, relieved by rest and sublingual GTN.

\section{Current Medications}

3 patients took Atenolol $50 \mathrm{mg}$ daily;

6 patients took Aspirin $100 \mathrm{mg}$ daily;

8 patients took daily Statins lipid lowering drug.

All patients continued their routine above-mentioned medications during receiving Moxa Tar treatment.

Frequency of angina pectoris attacks before treatment of Moxa Tar: from every one week to every three months. The average frequency of angina attacks of these 15 patients is one episode every one month.

\section{Method of treatment:}

Applying Moxa smoke to the precordia and the back of the heart for 15 minutes, one session a day, 15 days is a course. There is a 3-day interval between courses.

\section{Duration of treatment:}

All 15 patients had 3 courses (45 days) of treatment.

Assessment of efficacy of treatment of angina pectoris with Moxa Tar is based on the frequency of angina attacks:

After 3 courses of treatment 2 of the 15 patients had no improvement in the frequency of their angina attacks in the next 12 months.

2 of 15 patients had no angina attack in the next 12 months.

11 of 15 patients had significant improvement in the frequency of their angina attacks in the next 12 months. The frequency of angina attack of these 11 patients was reduced from one episode every month before treatment to one episode every 4 months after treatment. 
The response rate: $86.6 \%$

Failure rate: $13.4 \%$

The above figures indicate that Moxa Tar could effectively treat atherosclerosis.

Other moxibustion practitioners also report that moxibustion can significantly reduce the frequency and severity of angina.

\section{The treatment of diabetic foot with ulcers}

The author used Moxa smoke to treat 10 patients (8 patients in China, 2 patients in Australia) of diabetic foot with ulcers in their feet or lower legs which looked dark red and felt cold. The author applied Moxa smoke to their lower legs and feet with sparing the ulcers (Moxa Tar is a strong immune suppressor, directly applying Moxa smoke to ulcer may cause infection) for 15 to 20 minutes, one session a day, 15 days course with 3 to 5 days interval between courses. 3 patients had infection in their ulcers; they were treated with antibiotics at the same time. After 2 to 5 courses of treatment with Moxa smoke, 9 of the 10 patients' ulcers in the feet or legs were healed; the colour and the temperature of the legs and feet returned to normal. The author hypothesises that the microcirculation of the affected legs and/or feet has improved after the treatment with Moxa smoke.

Success rate: $90 \%$

Failure rate: $10 \%$

These observational evidences also indicate that Moxa Tar could treat atherosclerosis induced blood vessel disease.

Other moxibustion practitioners also report that moxibustion can effectively treat diabetic foot and diabetic ulcer too.

\section{Treatment of chronic renal failure due to aging}

One moxibustion practitioner reports that moxibustion can significantly improve the renal function of aged people who have chronic renal failure but without other kidney diseases. The cause of chronic renal failure in aged people who do not have other kidney diseases is due to renal artery atherosclerotic plaque formation when they become old. The atheroma narrows the lumen of renal artery and reduces renal blood flow, resulting in decreased glomerular filtration rate and chronic renal failure. The deteriorated renal function in these patients (elevated creatinine level) can indirectly reflect the severity of renal artery atherosclerosis. In Western medicine, no drug or therapy can treat chronic renal failure which is due to aging. That moxibustion can reverse chronic renal failure due to aging supports that Moxa Tar could treat atherosclerosis.

All the above observational evidences support that Moxa Tar could treat atherosclerosis, but so far there are no pathological evidences to prove that Moxa Tar can treat atherosclerosis. The author recommends that pathologists use animal model of atherosclerosis to test the therapeutic effect of Moxa Tar for treating atherosclerosis. Moxa Tar could be the solution to atherosclerosis as it is the solution to chronic laryngitis and other chronic inflammatory diseases.

\section{Discussion of the mechanism of Moxa Tar}

It is reported that moxibustion also can effectively treat or cure many other chronic inflammatory diseases, including thromboangiitis obliteran (Buerger's disease), Crohn's disease, ulcerative colitis, infertility secondary to chronic inflammatory pelvic disease, asthma, psoriasis, pulmonary fibrosis, Sarcoidosis, radiation colitis, radiation pneumonitis, recalcitrant migraine (it is due to chronic inflammation in the hypothalamus) and so on [11].

The author notices that Moxa Tar (Moxa smoke) can only treat chronic inflammatory diseases in which there are $\mathrm{T}$ cell infiltrates. Moxa Tar is not effective in treating hay fever, rheumatoid arthritis, or other immune complex induced inflammatory diseases. The underlying mechanisms in these conditions are type I and type III immune reactions. Immune complexes can directly activate neutrophils and complement system to trigger inflammatory reactions without the need of $\mathrm{T}$ cell involvement. The author therefore assumes that the active anti-inflammatory ingredients in Moxa Tar can inhibit $\mathrm{T}$ cell, molecules related to $\mathrm{T}$ cell activity or $\mathrm{T}$ cell products, resulting in blocking or terminating the chronic inflammatory reaction [4].

The author hypothesizes that Moxa Tar has potential to treat any $\mathrm{T}$ cell involved chronic inflammatory diseases. If its active antiinflammatory components can be isolated and purified to make a new drug, it will definitely revolutionize the treatment of chronic inflammatory diseases.

\section{Suggested Future Line of Research}

The author proposes the following future line of research:

Using animal chronic inflammatory models to test whether Moxa Tar has pathological evidence of anti-inflammatory effect and curative effect for glucocorticoid steroid resistant chronic inflammation. Possible options of animal inflammatory models include: mouse COPD model, mouse asthma model, Bleomycin induced mouse pulmonary fibrosis model and rabbit atherosclerosis model.

If animal inflammatory model test can confirm Moxa Tar has curative effect for glucocorticoid steroid resistant inflammation, the next step is to use the same animal inflammatory model to test whether Moxa-Tar-dissolved-water has curative effect for glucocorticoid steroid resistant inflammation (the author has used Moxa-Tar-dissolved-water to cure chronic laryngitis, glucocorticoid steroid resistant eczema and other chronic inflammatory diseases. This indicates that Moxa- Tar-dissolved-water contains the active antiinflammatory ingredients, but its potency of anti-inflammatory effect is much weaker than that of Moxa Tar).

If animal inflammatory model test can also confirm that Moxa-Tardissolved-water has curative effect for glucocorticoid steroid resistant inflammation, it will prove that Moxa-Tar-dissolved-water contains the active anti-inflammatory ingredients; therefore the active antiinflammatory ingredients of Moxa Tar could be isolated from it. The following is the possible method of isolation:

Moxa-Tar-dissolved-water can be separated into two different fractions by liquid chromatograph preparation. Then the antiinflammatory effect of each fraction can be tested separately on an animal inflammatory model. Theoretically only one part of the two separated fractions should contain the active anti-inflammatory components of Moxa Tar and show the positive anti-inflammatory result on animal test. Therefore it needs to eliminate the fraction which does not show anti-inflammatory effect on animal test and to proceed with the same separation and animal test on the positive fraction round-by-round to narrow down the active anti-inflammatory ingredients of Moxa Tar until they are eventually isolated and identified [10]. 
This work needs collaboration of organic chemist, pathologist and or pharmacologist. It can only be done in the university, research institute or drug company which has the facilities and funds.

Clinicians can do small group clinical trial using Moxa Tar (Moxa smoke) to treat eczema, COPD, chronic gastritis, angina, diabetic food, chronic sports injury and use more objective tests (including pathology) to assess whether Moxa Tar has curative anti-inflammatory effect. It is hard for people to believe that Moxa Tar has such unique and potent anti-inflammatory effect, but it is easy to prove it by clinical experiments or laboratory tests.

Immunologist can do laboratory experiments to see whether Moxa Tar can inhibit $\mathrm{T}$ cell activity and interfere cell-mediated immune reaction.

\section{Conclusion}

Moxa Tar has unique and potent anti-inflammatory property, which is different from that of glucocorticoid steroid. It can treat and cure glucocorticoid steroid resistant chronic inflammation. It is a cure of chronic non-specific inflammation.

It has been proved at clinical level that Moxa Tar is a cure for chronic laryngitis, chronic muscle and soft tissue injuries and a wide range of other incurable chronic inflammatory diseases. Moxa Tar could be a cure for the inflammation in COPD

Moxa Tar is an important source for developing new antiinflammatory drug.

\section{Epilogue}

A lot of efforts have been made to develop new anti-inflammatory drugs by researchers, but so far there has been no break-through. It is very difficult to find a new way of developing new anti-inflammatory drugs. Though Moxa Tar has such "magic" anti-inflammatory effect, it is little known to Western health professionals. The author could not overemphasize the significance of the possible role of Moxa Tar in conquering many glucocorticoid steroid resistant chronic inflammatory diseases (especially atherosclerosis and multiple sclerosis) and he hopes that it will open up a whole new field for health researchers (pathologists, immunologists, pharmacologists and clinicians) to develop new anti-inflammatory drugs or therapies.

\section{End Notes}

Meridian line: The term "meridian lines" is used in a number of natural therapies with acupuncture being perhaps the best known.
Meridian lines are invisible lines that carry energy throughout the body. Along these lines are points called meridian points. Meridian lines cannot be seen or felt like other systems in the body such as the circulatory or nervous system. When a person is in good (balanced) health, their meridian lines will be open and clear of blockages.

\section{References}

1. V Kumr, Al Abbas, N Fausto, R Mitchell, C. Alpers, et al. (2007) Robbins Basic Pathology Saunders, an imprint of Elsevier Inc, Philadelphia, PA.

2. R. Rubin, D Strayer (2008) Rubin's Pathology, clinicopathologic Foundations of Medicine. (5th edtn). Wolters Kluwer, Philadelphia, USA.

3. Fauci A, Braunwald E, Kasper D, Hauser S, Longo D (2008) Harrison's Principles of Internal Medicine. (17th edtn). McGraw-Hill Companies, New York, United States.

4. Li R, Li WJ, Cai YN, Li ZG, Luo Q, et al. (2005) Effects of Moxibustion at FeiShu and Gaohuang on expression of TGF-betal in the Bleomycininduced pulmonary fibrosis. Zhongguo Zhen Jiu 25: 790-792.

5. Dykewisz MS (2001) Newer and alternative non-steroidal treatment for asthmatic inflammation. Allergy Asthma Proc 22: 11-15.

6. Frew AJ, Plummeridge MJ (2001) Alternative agents in asthma. J Allergy Clin Immunol 108: 3-10.

7. Legg J, Warner J (2000) Asthma the changing face of drug therapy. Indian J Pediatric 67: 147-153.

8. Ribas DC, Monte CR, Garcia BM, Besoli MA, Soler MC (1999) Efficacy of methotrexate in the treatment of corticosteroid dependent asthmatic patients. Rev Clin Esp199: 142-146.

9. Kazimierezak A, Maziarka D, Skorupa W, Kus J (1997) Use of methotrexate for treatment of corticosteroid-dependent asthma. Pneumonol Alergol Pol 65: 225-230.

10. Aoki F, Kurabayashi M, Hasegawa Y, Kojima I (2005) Attenuation of bleomycin-induced pulmonary fibrosis by follistatin. Am J Respir Crit Care Med 172: 713-720.

11. Aono Y, Nishioka Y, Inayama M, Ugai M, Kishi J, et al. (2005) Imatinib as a novel antifibrotic agent in bleomycin-induced pulmonary fibrosis in mice. Am J Respir Crit Care Med 171: 1279-1285.

12. Arai T, Abe K, Matsuoka H, Yoshida M, Mori M, et al. (2000) Introduction of the interleukin-10 gene into mice inhibited bleomycininduced lung injury in vivo. Am J Physiol Lung Cell Mol Physiol 278: L914- L922.

13. Arslan SO, Zerin M, Vural H, Coskun A (2002) The effect of melatonin on bleomycin-induced pulmonary fibrosis in rats. J Pineal Res 32: 21-25.

14. Kumar V, Abbas A, Fausto N, Mitchell R, Alpers C, et al. (2007) Robbins Basic Pathology, (8thedn), Saunders, Philadelphia, PA, USA, pp: 343-353.

15. http://accessmedicine.mhmedical.com/book.aspx?bookId=331.

16. Wang Q, Wang Y, Hyde DM, Gotwals PJ, Koteliansky, et al. (1999) Reduction of bleomycin induced lung fibrosis by transforming growth factor beta soluble receptor in hamsters. Thorax 54: 805-812. 